\title{
Neural Network based classification of Bio Optical signals for detection of cell abnormalities of human skin
}

\author{
G.Subramanya Nayak \\ Department of E \&C Engineering, \\ Manipal Institute of Technology \\ Manipal University \\ Manipal -576104
}

\author{
Puttamadappa C \\ Department of E \&C Engineering \\ SJB Institute of Technology \\ Uttarahalli Road, Kengeri \\ Bangalore -60
}

\begin{abstract}
Early diagnosis of precancerous and malignant lesions is critical for the improving of the current poor survival rate of patients with a variety of tumors. The development of new high-specificity and high-sensitivity imaging technologies can play an important role in the early diagnosis, accurate staging, and treatment of cancer. Bio-optical signals are the result of the optical functions of the biological systems, occurring naturally or induced by the measurement. The identification of the state of human skin tissues is discussed here. The Bio-optical signals recorded in vitro have been analyzed by extracting various statistical features. Using MATLAB programs, various statistical features are extracted from both normal and pathology spectra. Different features like mean, energy etc were extracted. The values of the feature vector reveal information regarding tissue state. Then a classical multilayer feed forward neural network with back propagation algorithm is employed to serve as a classifier of the feature vector, giving $100 \%$ successful results for the specific data set considered. Furthermore, a small time needed to acquire and analyze the fluorescence spectra together with high rates of success, proves our method very attractive for real time applications.
\end{abstract}

\section{Keywords}

Neural Networks, Back Propagation algorithm

\section{INTRODUCTION}

The cancer is one of the leading causes of death all over the world, but, if detected early, can be curable. In the current study, the data analysis and classification of pathological conditions of the optical spectra of skin cancer are performed using MATLAB programs. The proposed study is done on MITI-FOPTO database. A large amount of data needs to be analyzed for the classification of normal and pathology conditions. Using MATLAB functions, features like differentiation and count of spikes for different thresholds, mean, standard deviation, energy, residuals on curve fitting, LPC coefficients were extracted. These are used for the classification of spectra in normal and pathological states, using Artificial Neural Network.

Before feature extraction, the signals are normalized and then filtered to eliminate undesirable spikes due to noise and other disturbances.
The $10^{\text {th }}$ degree polynomial curve is fitted onto pathology spectra as shown in Figure 1.

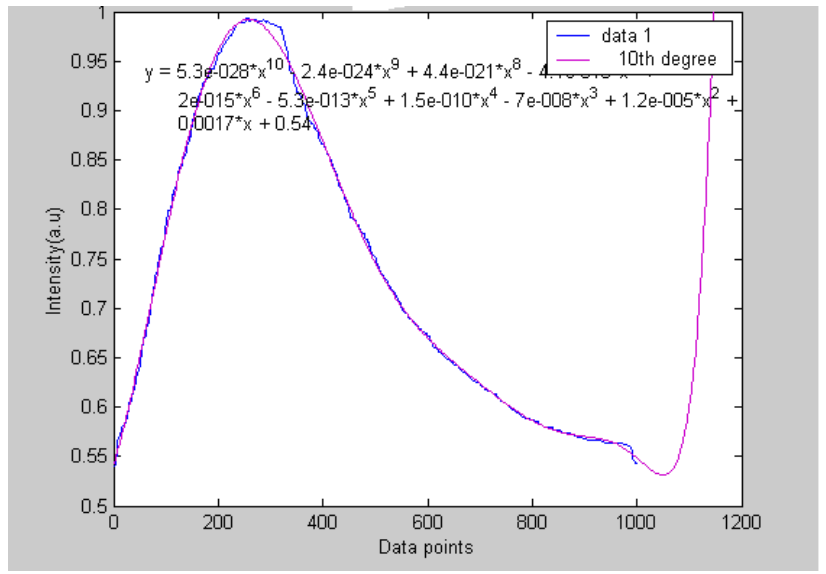

Figure 1. Curve Fitting onto Optical Spectra

This paper deals with the data analysis and classification of pathological conditions of the optical spectra of skin cancer performed using MATLAB.

\section{ANN DIAGNOSTIC ARCHITECTURE}

An ANN structure is employed for the classification of spectra. It consists of four modules as shown in Figure 2.

The signals are filtered with 21 ordered median filters to eliminate undesirable spikes due to noise and other disturbances. The signal is then fed to the feature extraction module and different features were extracted.

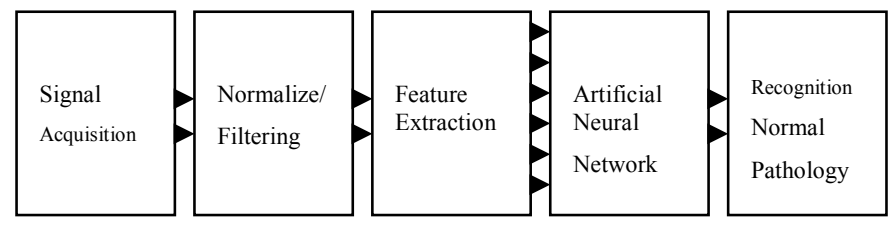

Figure 2. The Model scheme used to perform diagnosis 


\section{FEATURE EXTRACTION 1) Differentiation}

DIFF (X) for a vector $X$ is [X (2)-X (1) X (3)-X (2) ... X (n)-X (n$1)]$. DIFF $(X)$ for a matrix $X$ is the matrix of row differences, $[X$ $(2: \mathrm{n},:)-\mathrm{X}(1: \mathrm{n}-1,:)][13,14] .1^{\text {st }}$ order differentiation was carried out on each normal and pathology spectra.

After completion of finding the number of peaks at thresholds 100, 250, 500, 750 for 25 normal and 25 pathology spectra, the mean, standard deviation and variance were determined and are tabulated in Table 3.1 and Table 3.2 .

Table 3.1: Number of peaks in normal spectra

\begin{tabular}{|c|c|c|c|}
\hline Threshold & Mean & Std.deviation & Variance \\
\hline $\mathrm{TH}>750$ & 4.045 & 3.966 & 9.6193 \\
\hline & & & \\
\hline $\mathrm{TH}>500$ & 14.5 & 7.8606 & 97.2143 \\
\hline & & & \\
\hline $\mathrm{TH}>250$ & 112.1823 & 44.8367 & $1.937 \times 10^{3}$ \\
\hline & & & \\
\hline $\mathrm{TH}>100$ & 487.0455 & 62.8906 & $4.7 \times 10^{3}$ \\
\hline
\end{tabular}

Table 3.1: Number of peaks in arrhythmia spectra

\begin{tabular}{|c|c|c|c|}
\hline Threshold & Mean & Std.deviation & Variance \\
\hline $\mathrm{TH}>750$ & 4 & 2.7134 & 2.5714 \\
\hline & & & \\
\hline $\mathrm{TH}>500$ & 21.7273 & 4.8617 & 23.6364 \\
\hline & & & \\
\hline $\mathrm{TH}>250$ & 178.3636 & 20.037 & 401.4805 \\
\hline & & & \\
\hline $\mathrm{TH}>100$ & 551.8182 & 24.189 & 585.1082 \\
\hline
\end{tabular}

\section{2) Energy}

For vectors, SUM (X) is the sum of the elements of $\mathrm{X}$, for matrices, SUM (X) is a row vector with the sum over each column that corresponds to energy.

The energy was obtained for different windows of data points for normal and pathology spectra where significant variations in energy distributions were observed.

\section{3) $4^{\text {th }}$ order linear prediction coefficients (LPC)}

$4^{\text {th }}$ order linear prediction coefficients for normal and pathology spectra are determined and are found as one of the prominent discriminating factor between these two cases. The coefficients like radius and angle are the features that are obtained as per the equation given below.

The radius and the angle in radians are taken from the polar diagram.

\section{ANN IMPLEMENTATION}

\section{A. Design of ANN with Back Propagation Algorithm:}

Three layer networks are sufficient to design any nonlinear network. Input is a layer of nodes with three feature vectors as input. Single second and third layers have same activation function for all neurons. Activation function is a tan sigmoid function as shown in Figure 3.

LM Back propagation algorithm, is used for training.

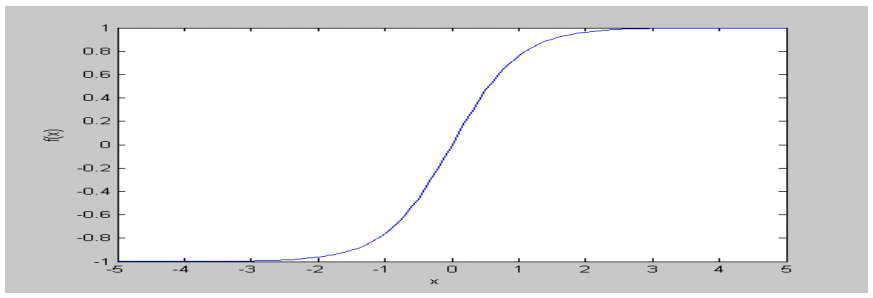

Figure 3. Activation function - Tan sigmoid

\section{B. Training of ANN:}

Data from normal and pathology were obtained from data base. Data of 40 normal and 40 pathology are used to train the network.

Feature vector is constructed and training program is executed. The training of ANN stops once the performance goal is met, as shown in Figure 4. The convergence is achieved in 262 epochs.

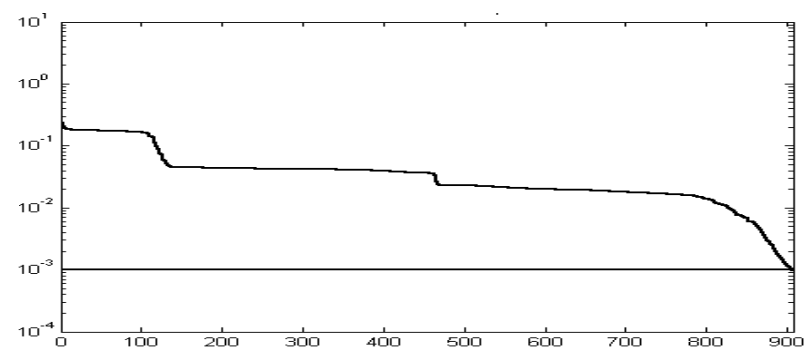

Figure 4. Training of ANN 


\section{RESULTS}

Feature vector of 25 test data of Normal and 25 test data of pathology are given to network to classify. The results are shown in Table 5.1 .

Table 5.1 Classification results

\begin{tabular}{|c|c|c|c|}
\hline $\begin{array}{l}\text { Test } \\
\text { data }\end{array}$ & $\begin{array}{c}\text { Desired } \\
\text { output }\end{array}$ & $\begin{array}{c}\text { Classifier } \\
\text { output }\end{array}$ & $\begin{array}{c}\text { Output } \\
\text { Characterization }\end{array}$ \\
\hline N1 & 1 & 1.0000 & Normal \\
\hline N2 & 1 & 1.0000 & Normal \\
\hline N3 & 1 & 1.0000 & Normal \\
\hline N4 & 1 & 1.0000 & Normal \\
\hline N5 & 1 & 1.0000 & Normal \\
\hline N6 & 1 & 1.0000 & Normal \\
\hline N7 & 1 & 1.0000 & Normal \\
\hline N8 & 1 & 1.0000 & Normal \\
\hline N9 & 1 & 1.0000 & Normal \\
\hline N10 & 1 & 1.0000 & Normal \\
\hline N11 & 1 & 1.0000 & Normal \\
\hline N12- N25 & 1 & 1.0000 & Normal \\
\hline P1 & -1 & -0.9923 & Pathology \\
\hline P2 & -1 & -0.9008 & Pathology \\
\hline P3 & -1 & -0.8025 & Pathology \\
\hline P4 & -1 & -0.9078 & Pathology \\
\hline P5 & -1 & -0.8070 & Pathology \\
\hline P6 & -1 & -0.1000 & Pathology \\
\hline P7 & -1 & -0.8970 & Pathology \\
\hline P8 & -1 & -0.8033 & Pathology \\
\hline P9 & -1 & 0.9776 & Pathology \\
\hline P10- P15 & -1 & -0.9880 & Pathology \\
\hline P16 & -1 & 1.0000 & Normal \\
\hline P17 - P24 & -1 & -0.9008 & Pathology \\
\hline P25 & -1 & 1.0000 & Normal \\
\hline
\end{tabular}

\section{CONCLUSION}

Our method achieves the discrimination between normal and pathology optical spectra. Furthermore, a small time needed to acquire and analyze the spectra together with the high rates of success, proves our method very attractive for real -time applications. The results of ANN has been verified with the results of PCA and found satisfactory. This gives us to firmly decide on the pathology. Further, performance parameters can be accurately judged if the tests are carried out on large number of spectra. As less number of features is used, computational delay in training the ANN is reduced. Gradient descent algorithm is too slower and convergence depends on learning rate. Hence high performance algorithm (Levenberg - Marquardt algorithm) which operates in batch mode is used for fast training. Specificity, Sensitivity and Accuracy obtained are 100\%, $92 \%$ and $96 \%$ respectively.

\section{REFERENCES}

[1] Jacek M. Zurada , Introduction to Artificial Neural Systems,pp 25-89. Publisher-Pearson Education, 3rd Edition.

[2]B.K.Manjunath,J.Kurien,C.Muralikrishna,Autofluorescence of Oral tissue for optical pathology in Oral malignancy, Journal of Photochemestry and Photobiology B. Biology 73 (2004 ) 49 -58.

[3] George A.Rovithakis and Michail Maniadakis ,Artificial Neural Networks for discriminating Pathologic from Normal Peripheral Vascular Tissue,IEEE transactions on Biomedical Engineering ,vol 48,No 10, october 2001

[4] Sigurdur Sigurdsson et ,al ,Detection of skin cancer by classification of Raman spectra, IEEE transactions on Biomedical Engineering

,2004.

[5] Simon Hykin,Neural Networks-A Comprehensive foundation, section 4.1to4.10,page 156-234,Jaico Publishing House, $5^{\mathrm{TH}}$

edition.

[6] William Palm J, "Introduction to MATLAB 6 for Engineers".

[7]G.S.Nayak, Sudha Kamath et al. (2006) Principal Component Analysis and Artificial Neural Network Analysis of oral Tissue Fluorescence Spectra: Classification of Normal, Premelignant and Malignant Pathological Condition Biopolymers 82:152-166

[8] G.Subramanya Nayak and Gopallakrishna Prabhu K (2004) Analysis of Electroglottographic Signal for Measurement of Vocal Fold Abduction / Larynx Movement ,using Amplitude Modulation Technique,ECCS-2004 Proc. National Conference on Electronic Circuits and Communication Systems, Patiala, India, 2004, pp $463-46$ 